\title{
Analytical Performance Model for FPGA-based Reconfigurable Computing
}

\author{
Hossein Mehri and Bijan Alizadeh* \\ School of Electrical and Computer Engineering, College of Engineering, University of Tehran, Iran
}

\begin{abstract}
Traditionally, FPGA designers make use of CAD tools for evaluating architectures in terms of the area, delay and power. Recently, analytical methods have been proposed to optimize the architectures faster and easier. A complete analytical power, area and delay model have received little attention to date. In addition, previous works use analytical methods to optimize general-purpose FPGA architectures. Using analytical models for optimizing application-specific FPGA architectures are interesting subjects in the reconfigurable computing field. In this way, designers can investigate the optimized architecture for a set of application circuits and the consumers can find their best architecture among a variety of devices which is optimal for their specific work. In this paper, we complete an analytical FPGA performance model by presenting an analytical model to estimate the dynamic and leakage power and by integrating it into the Geometric Programming framework. This way, we are able to rapidly analyze various FPGA architectures and select the best one in terms of power consumption as well as area and delay. In the next step, we extend the model for optimizing FPGA architectures for a set of applications. A case of the best architecture for two specific circuits has been investigated in this paper.
\end{abstract}

Keywords: Analytical models, general-purpose FPGA architectures, application-specific FPGA architectures, power, reconfigurable computing

\section{INTRODUCTION}

Need for flexibility forced designers to Field Programmable Gate Arrays (FPGAs) against Application Specific Integrated Circuits (ASICs). However, FPGAs are not power efficient because they consist of a large number of interconnects and programmable switches and also generic logic structures in FPGAs consume more power than the dedicated circuitry in ASICs. Hence, managing the power by means of architectural optimization has emerged as one of the most important FPGA-based design challenges. Traditionally, designers make use of Computer-aided Design (CAD) tools to evaluate architectures in terms of the area, delay and power. This way, benchmark circuits are mapped into architectures under investigation using CAD tools which is a time consuming and expensive task. Recently, analytical methods have been proposed in which FPGA architectures are modeled by simple equations [16]. Then by feeding analytical models to optimization tools designers will be able to optimize the architectures faster and easier.

On the other hand, most analytical methods proposed up to now, are able to model the area and delay [6-9]. The authors in [10-13] have presented CAD tools to estimate FPGA power dissipation. Although [14-15] have tried to model leakage power analytically, a complete power model to cover both dynamic power and leakage power together is not proposed yet. Please note that both static and dynamic powers are critical elements in the power calculation. FPGA vendors are trying to provide viable low-power devices, but as process technologies shrink from $130 \mathrm{~nm}$ to $90 \mathrm{~nm}$ to $65 \mathrm{~nm}$ and so on, transistors become inherently more leaky and therefore static power consumption rises. Furthermore, the extremely high performance requirements of systems using FPGAs drive dynamic power consumption up, a function of the frequency and the number of switching nodes.

The main problem with power model for FPGAs is that the power widely depends on inputs and configuration bits which maintain circuit's behavior. In [15] the authors proposed a state dependent analytical leakage power

\footnotetext{
* Corresponding author at School of Electrical and Computer Engineering,

College of Engineering, University of Tehran, Tehran, 14395-515, Iran.

E-mail addresses: h.mehri@ut.ac.ir (H. Mehri), b.alizadeh@ut.ac.ir (B. Alizadeh).
} 
model for FPGA architectures. The model discusses subthreshold leakage power and gate leakage power in FPGAs. Reverse biased junction band-to-band-tunneling leakage (BTBT) power, is not taken into account and the model does not take the dynamic power into account. In [16], the authors indicated that energy can be defined as a function of routed wire length and critical path delay. Therefore, they used existing wire length and critical path delay models to derive an analytical energy model for FPGAs. The model just takes into account the routing power as a linear function of routed wire length.

In [10], the authors proposed a detailed power consumption model for FPGA architectures. The model has been added into the Versatile Place and Route (VPR) framework [21] and provides a tool to evaluate different architectural impacts on power dissipation within FPGA architectures. This VPR power model can be used as an experimental power estimation CAD tool for FPGAs.

The authors of [6] proposed an area and delay model for FPGAs which combines high-level and low-level architecture parameters. The authors used Geometric Programming (GP) to optimize the architecture. This framework allows high-level architecture parameter selection and transistor sizing to be done concurrently. The model has been validated for general-purpose FPGA architecture optimizations in comparison with CAD tools based experimental methods. The authors of [24] used two representative architecture questions to provide an understanding of the capabilities and the limitations of the analytical models. The paper justifies the use of the analytical models to optimize general-purpose FPGA architectures as a capability and shows the limitation of analytical models that cannot capture the behavior of some applications that contain discrete effects. The experiments of the paper are based on using one circuit for an application-specific FPGA architecture.

The authors of [25] proposed application specific inflexible FPGA (ASIF) which is an FPGA with reduced flexibility that implements a set of application circuits which will operate at mutually exclusive times. The idea has been extended to Heterogeneous-ASIF by [26] which is an ASIF reduced from a heterogeneous FPGA. The work is based on removal approach to inflexible FPGA and only the area optimization is considered.

This paper describes a power model that estimates the dynamic and leakage power for a wide variety of FPGA architectures analytically. This power model has been integrated into the GP framework. This paper also investigates the impact of various architectural parameters on the power-efficiency of FPGAs. Then the model integrated into GP performance model using area-delay model proposed by [6]. After validating the GP performance model, the model has been extended to optimize application-specific FPGA architectures. This model helps to explore the architectures for FPGA-based reconfigurable computing purposes. In summary, the main contributions of this paper are as follows:

- Analyzing the dynamic power and leakage power of an FPGA analytically using simple equations.

- Formulation of the power model as a geometric program and integrating it to a complete FPGA performance model using existing area-delay models.

- Extending the proposed GP performance model to optimize application-specific FPGA architectures for reconfigurable computing purposes.

This paper is organized as follows. Section 2 gives a brief review of FPGA internal architecture, area model, delay model and geometric programming. Section 3 describes our proposed power model and the model developed in the multi-application framework in Section 4. In order to measure the accuracy of the proposed models, the experimental results are shown in Section 5. Finally, a brief conclusion is presented in Section 6.

\section{Preliminaries}

\subsection{FPGA Internal Architecture}

Common FPGAs used by commercial vendors have mesh-based architectures as shown in Fig. 1 [17]. The logic part of architecture to implement the function is integrated into configurable logic blocks (CLBs). The CLBs (or clusters) are arranged on a grid and are interconnected by a programmable routing network. Each CLB consists of $\mathrm{N}$ basic logic elements (BLEs). In this way, $\mathrm{N}$ is called cluster size. Each BLE consists of a $\mathrm{K}$-input Lookup-table (KLUT), a register and a multiplexer and some buffer. K is called LUT size. The structure of a CLB is shown in Fig. 2. The routing network consists of horizontal and vertical routing channel tracks. Switch box and connection box are two elements of routing network. Switch boxes connect horizontal and vertical routing tracks of the routing network. Connection boxes connect logic block pins with adjacent routing tracks. A summary of model parameters including 
architectural parameters (under investigation) and circuit parameters (e.g. $n_{2}$ which is the number of LUTs when the circuit is implemented in 2-input LUTs) is shown in Table 1.

\subsection{Area Model}

In order to make this paper self-contained, the analytical area model, which is used in this paper as a part of the GP performance model, is explained in more details in this subsection. This model is borrowed from [6]. The total area of an FPGA consists of logic area, $A_{l}$, and routing area, $A_{r}$, as equated in (1).

$$
A_{\text {total }}=A_{l}+A_{r}
$$

Logic area is represented as (2) where $N_{c}$ is the number of CLBs and $A_{C L B}$ is the area of each CLB.

$$
A_{l}=N_{c} A_{C L B}
$$

The area of a CLB is represented as (3) where $A_{L U T}$ is the area of a LUT, $A_{\text {reg }}$ is the area of a register, $A_{21 m u x}$ is the area of 2:1 multiplexer, $A_{L S m u x}$ is the area of the LUT input select multiplexer, $B_{l o}$ is the area consumed by CLB output buffer and $A_{c l k B}$ and $A_{r s t}$ are the area consumption of the clock buffer and set/reset logic. Note that, their areas are represented as the sum of the areas of their comprised transistors.

$$
A_{C L B}=N A_{L U T}+N A_{\text {reg }}+N A_{21 m u x}+K N A_{L S m u x}+N B_{l o}+A_{c l k B}+A_{r s t}
$$

The routing area is also the sum of connection boxes areas and switch boxes areas as shown in (4). These areas are detailed in [6].

$$
A_{r}=A_{C B}+A_{S B}
$$

\subsection{Delay Model}

The analytical delay model borrowed from [6] is explained in this subsection. The delay model models the critical path delay of the circuit implemented on FPGA. The critical path will emanate from a register and typically passes through a number of LUTs, CLBs, switch boxes, connection boxes, CLB feedback paths and the multiplexers and buffers associated with them. This way, the critical path delay is represented as (5) which is the weighted sum of components' delays. The weights depend on the expected number of each component. The depth of the netlist when implemented in K-input LUTs is represented by $D_{k}$. The number of CLBs through which the critical path travels is represented by $D_{c}$. The number of internal feedback connections through which the critical path travels is represented by $D_{i}$. The number of switch boxes through which each external connection on the critical path propagates is represented by $D_{r}$. $T_{\text {reg to oDrv }}$ is the delay from the register producing the critical path signal through the multiplexer selecting whether the LUT output is registered or not, and through the two-level inverting driver. The term $T_{L U T F / B}$ path is the delay from the basic logic element output buffer through the pass transistor-based MUX on the LUT input to its buffer. $T_{L U T}$ delay is the delay from the LUT driver through all levels of the multiplexer implementing the LUT, 2:1 select MUX and to the LUT output driver. The term $T_{O / P C B \text { delay }}$ is the delay from the logic element's output buffer, through the switch box multiplexer to its first inverting buffer where in this case the Elmore delay is through the path to the switch box driver. $T_{S B}$ delay is the routing path signal between switch boxes. This is represented by the sum of the driver delay and delay through the two-level switch box multiplexer. The term $T_{I / P C B}$ delay is the path through the switch box to the connection box where the routed signal is consumed. This is the sum of the initial driver delay, the delay through the connection box multiplexer and through the two inverting drivers in the connection box. $T_{\text {input }}$ MUX delay is the delay from the connection box output driver through the LUT input select multiplexer to the LUT input driver. The term $T_{L U T}$ to reg delay is the delay through the LUT driver, then the multiplexer implementing the LUT and to the register input, where the critical path terminates. Each component's delay can be calculated by representing the component as its RC network model and then using the Elmore delay model. For example, (6) is the constraint which is representing the delay from the logic element's output buffer, through the switch box multiplexer to its first inverting buffer [6]. 


$$
\begin{aligned}
& T_{\text {total }}=T_{\text {reg to oDrv }}+D_{i} T_{L U T \text { F } / B \text { path }}+\left(D_{k}-1\right) T_{L U T \text { delay }}+D_{c} T_{O / P C B \text { delay }}+D_{c} D_{r} T_{S B \text { delay }}+D_{c} T_{I / P C B \text { delay }} \\
& +D_{c} T_{\text {input MUX delay }}+T_{L U T} \text { to reg delay } \\
& T_{O / P C B \text { delay }} \geq R_{D, n, L O d r v 2}\left(C_{D, n, L O d r v 2}+N K C_{D, L S m u x}+F_{c, \text { out }} C_{D, S B \text { mux }}\right)+\left(R_{D, n, L O d r v 2}+R_{D, S B \text { mux }}\right) 2 C_{D, S B \text { mux }} \\
& +\left(R_{D, n, L O d r v 2}+2 R_{D, S B \text { mux }}\right) \times\left(C_{D, S B \text { mux }}+C_{G, S B d r 1}\right)
\end{aligned}
$$

\subsection{Geometric Programming}

A geometric program (GP) is a type of mathematical constrained optimization problem characterized by constraint and objective functions that has a special form as follows:

$$
\begin{array}{ll}
\text { Minimize: } & \mathrm{f}_{0}(\mathrm{x}) \\
\text { Subject to: } & \mathrm{f}_{\mathrm{i}}(\mathrm{x}) \leq 1, \text { for } \mathrm{i}=1,2, \ldots, \mathrm{m} ; \\
& \mathrm{g}_{\mathrm{i}}(\mathrm{x})=1, \text { for } \mathrm{i}=1,2, \ldots, \mathrm{n} ;
\end{array}
$$

where $\mathrm{x}$ is a strictly positive $\mathrm{n}$-vector of real values, and $\mathrm{f}_{0}, \ldots, \mathrm{f}_{\mathrm{m}}$ are posynomials and $\mathrm{g}_{0}, \ldots, \mathrm{g}_{\mathrm{n}}$ are monomials. A monomial is defined as a function $g(x)=c x_{1}{ }^{a_{1}} x_{2}{ }^{a_{2}} \ldots x_{n}{ }^{a_{n}}$ where the coefficient $\mathrm{c}$ must be strictly positive. A posynomial is simply a sum of a finite number of monomials. It should be noted that the restriction to strictly positive leading coefficients in a monomial is a serious one and explicitly disallows many problems, including general polynomial optimization rather than posynomial optimization.

Recently developed solution methods are able to solve even large-scale GPs extremely efficiently and reliably; at the same time a number of practical problems, particularly in circuit design, have been well approximated by GPs. This way, we can get effective solutions for the practical problems. The basic approach in GP modeling is to attempt to express a practical problem, such as a design problem, in GP format. In the best case, this formulation is exact; when this is not possible, we settle for an approximate formulation. In [18], the reader can find out a review of GP in the context of circuit design.

\section{Proposed Performance Model based on Geometric Programming}

In this section we explain our proposed GP performance model; consists of area, delay and power models. The main focus of this section is on describing a power model, while existing area and delay models described in [6] are used. This power model allows us to analyze the power dissipation at the transistor level for each component inside the FPGA. The average power consumption in digital circuits is broken down to three parts: dynamic, short-circuit and leakage power. We ignore the short-circuit power dissipation in this work because: 1) it decreases in submicron technologies when the supply voltage gets closer to the threshold voltage; 2 ) it is modeled as a percentage of the dynamic power [10]. Hence, the total power of an FPGA, $P_{\text {total }}$, can be represented as the sum of dynamic power $P_{d y}$ and leakage power $P_{l k}$, as denoted by (7).

$$
P_{\text {total }}=P_{d y}+P_{l k}
$$

In the rest of this section we will describe how to estimate these components in order to efficiently perform architectural exploration using the GP framework.

\subsection{Dynamic Power}

Dynamic power is the dominant component of the total power in FPGAs. It is dissipated every time a signal changes due to the charging and discharging of load and parasitic capacitances. Therefore, dynamic power is closely related to the transition density of all nodes in the circuit. The total dynamic power dissipation can be written as follows:

$$
\text { Dy. Power }=\sum_{\text {all nodes }} 0.5 C_{\text {node }} V_{\text {supply }}^{2} D_{\text {node }} f_{\text {clk }}
$$

where $V_{\text {supply }}$ is the supply voltage, $D_{\text {node }}$ is the transition density at each node, and $C_{\text {node }}$ is the capacitance of each node that is charged and discharged during each transition. In other words, the dynamic power is equal to the energy per clock cycle multiplied by the clock frequency, $f_{c l k}$, which is bounded by the critical path delay of the circuit. 
In analytical methods, the transition density of signals within the FPGA depends on the circuit inputs and configuration bits programmed to SRAMs which determine the circuit behavior. The architectural investigation is completely independent of the circuit inputs. In other words, in order to optimize the performance in terms of the area, delay or power, we are trying to find out the best values for architectural parameters (e.g. answering this question that 4-LUTs is better to be selected or 5-LUTs?) while there is no information about the circuit inputs.

On the other hand, in experimental methods which are using CAD tools to map benchmark circuits to the architectures under investigation, the circuit functionality is taken into account to estimate the power. In [10] the activity generator estimates the transition density of all nodes based on the circuit functionality by propagating transition densities from the inputs to the outputs while the transition density of inputs is assumed to be 0.5 . In this paper, however, we would like to estimate the power analytically. For doing so, instead of calculating the transition density of circuit's nodes by propagating the transition density from the inputs to the outputs, we take the transition density parameter out of the decision and assume it is constant. Note that it is not a big assumption because the main goal in this work is to quickly explore possible architectures and find out the optimized one. Hence, although the power estimated by analytical methods may not be the same as the power estimated by experimental ones, the optimized architectures are the same.

As seen in (8), the dynamic power consumption in FPGAs is the sum of routing dynamic power, $P_{d y . r o u t e}$, and logic block dynamic power, $P_{d y . l o g i c}$. In the following subsections we will discuss how to compute $P_{d y . l o g i c}$ and $P_{\text {dy.route }}$.

$$
P_{d y}=P_{d y . l o g i c}+P_{d y . r o u t e}
$$

\section{Routing Dynamic Power.}

A key part of any FPGA is the routing fabric. The power consumption of routing fabric is shown to be a linear function of the routed wire-length [10,20] and therefore dynamic power consumption in the routing fabric is a major concern that should be taken into account. To model the routing dynamic power in terms of wire-length, we need to determine the related scaling factor. For doing so, we have carried out some experiments by means of VPR power model for $6 \mathrm{MCNC}$ circuits. On the other hand, the analytical model for pre-routed wire-length (WL) of FPGAs in [5] is employed to estimate the routing dynamic power. Fig. 3 shows the Davis/Lam, Feuer/Lam and Davis-UB wire-length models discussed in [5] that are scaled down to match with the routing dynamic power computed by the experimental data. Note that we have used geometric mean across all circuits for experimental and modeled data. Based on the data shown in Fig. 3, we have selected the WL model (Davis/Lam) which is closest to the experimental results. The model is as follows:

$$
W L=\frac{\frac{p-0.5}{p}-\sqrt{n_{c}}-\frac{p-0.5}{6 \sqrt{n_{c}}}+\frac{-p-1+4^{(p-0.5)}}{2 p(p+0.5)(p-1)}}{1+\frac{-2 p-1+2^{(2 p-1)}}{2 p(p-1)(2 p-3)} n_{c}{ }^{(p-0.5)}-\frac{p-0.5}{6 p \sqrt{n_{c}}}-\frac{(p-0.5) \sqrt{n_{c}}}{p-1}} * \frac{4 f_{\text {avg }}}{3+f_{\text {avg }}}
$$

where, $\mathrm{nc}$ is the number of CLBs from [4] which also dictates the size of grid, $\mathrm{p}$ is the Rent's parameter of the circuit and favg is the average fan-out that is computed based on the technique presented in [5].

\section{Logic Block Dynamic Power.}

Based on mesh-based FPGA architecture in Fig. 1, the logic block dynamic power, $P_{d y . l o g i c}$, is expressed by (9). The size of the FPGA is assumed to be the smallest square that fits the benchmark circuit. Thus, the grid size is $N_{c}=\left\lceil\sqrt{n_{c}}\right\rceil^{2}$, where $n_{c}$ is the number of CLBs in the benchmark circuit and can be estimated using the formula in [4]. $P_{d y . C L B}$ is the dynamic power of a CLB and is represented by (10).

$$
\begin{aligned}
& P_{d y . \text { logic }}=N_{c} P_{d y . C L B} \\
& P_{d y . C L B}=K N P_{\text {input.mux }}+N P_{\text {lut }}+N P_{\text {register }}+N P_{\text {output.mux }}+P_{\text {reset }}
\end{aligned}
$$

where $P_{\text {input.mux }}, P_{\text {lut }}, P_{\text {register }}, P_{\text {output.mux }}$ and $P_{\text {reset }}$ are the power dissipated in LUT input select multiplexer, in a Lookup-Table, in the register, in LUT output multiplexer and in set/reset logic block, respectively. 
In order to compute the power dissipated in different parts of CLBs, let us consider the internal structure of lookup-tables shown in Fig. 4. There are $1+2+\ldots+2^{k-1}$ internal nodes in a K-input LUT. To estimate the power dissipated in a multiplexer tree we need to estimate the capacitance of each node. Each node within the lookup-table is associated with three source/drain capacitances (CS/D) and one gate capacitance (Cgate) due to the Miller effect. The dynamic power of a K-input LUT, $P_{d y . l u t}$, is represented by (11).

$$
P_{\text {dy.lut }}=0.5\left(2^{k}-1\right)\left(3 C_{S / D}+C_{\text {gate }}\right) V_{\text {supply }}^{2} D_{\text {constant }} f_{\text {clk }}
$$

To model multiplexers, we make use of the two-level multiplexing scheme because it is more compatible with the GP. This would implement the multiplexing in two stages as shown in Fig. 5(a). The first and second level of multiplexing is balanced where each stage has multiplexers with the same number of inputs. Another choice is modeling multiplexers with 2-input multiplexers. The number of internal nodes in this case is from the order of $2^{n}$, where $\mathrm{n}$ is the number of multiplexer inputs. Please note that two to the power of an optimization parameter (n) is not allowed in the GP formulation unless $\mathrm{n}$ is a constant. The only logic parameters that are constant in each run of $\mathrm{GP}$ are $\mathrm{K}$ and $\mathrm{N}$ that are considered as inputs while other parameters need to be optimized concurrently.

An example of a 16:1 multiplexer using this scheme is shown in Fig. 5(a). Each of 4:1 Multiplexers can be implemented using pass transistors, as shown in Fig. 5(b). So the expression for n:1 multiplexer can be expressed as (12).

$$
P_{\text {dy.mux }}=0.5\left((n+\lfloor\sqrt{n}\rfloor) C_{S / D}+\frac{n+\lfloor\sqrt{n}\rfloor}{2} C_{\text {gate }}\right) V_{\text {supply }}^{2} D_{\text {constant }} f_{\text {clk }}
$$

The input multiplexers select the lookup-table input signals among the logic block inputs (I) and feedback path $(\mathrm{N})$, so such multiplexers have I+N inputs. LUT output multiplexers, which select if it is registered or not, are 2:1 multiplexers. Note that the dynamic power of the set/reset block is ignored because of low switching activity.

\subsection{Leakage Power}

By the scaling of technology toward $65 \mathrm{~nm}$ and beyond, leakage power becomes more important. Leakage was not a concern in earlier technologies but it cannot be ignored in sub $100 \mathrm{~nm}$ technologies. Three major parts of total leakage power are: 1) subthreshold leakage, 2) gate leakage and 3) reverse biased junction band-to-band-tunneling leakage (BTBT). Subthreshold leakage is caused by the current between source and drain when the gate voltage is smaller than threshold i.e. in weak inversion. Gate leakage is caused by the current flow from the gate to the channel. BTBT leakage is caused by tunneling of electrons from the n-type source/drain to p-type substrate in NMOS device in the presence of a very high electric field.

Similar to dynamic power, leakage power, $P_{l k}$, can be represented as the sum of logic and routing parts as expressed by (13) where Plk.logic and Plk.route are computed based on (14) and (16), respectively. $P_{l k . C L B}$ is the leakage power of a CLB and can be expressed by (15).

$$
\begin{aligned}
& P_{l k}=P_{l k . l o g i c}+P_{l k . r o u t e} \\
& P_{l k . l o g i c}=N_{c} P_{l k . C L B} \\
& P_{l k . C L B}=K N P_{l k . i n p u t . m u x}+N P_{l k . l u t}+N P_{l k . r e g i s t e r}+N P_{l k . o u t p u t . m u x} \\
& \quad+P_{l k . c l k . b u f f e r}+P_{l k . r e s e t}+K N P_{l k . m u x . b u f f e r}
\end{aligned}
$$

where $P_{l k . i n p u t . m u x}$ is the leakage power of input multiplexer and $P_{l k . l u t}$ is the leakage power dissipated in an LUT. $P_{l k . r e g i s t e r}$ is the leakage power of LUT output register and $P_{l k . o u t p u t . m u x}$ is the leakage power of output multiplexer. $P_{l k . c l k . b u f f e r}$ is the power dissipated in the buffers of clock system and $P_{l k . r e s e t}$ is the leakage power of the set/reset block and $P_{l k . m u x . b u f f e r}$ is the power dissipated in the buffer of input multiplexers.

We need to calculate the leakage power of the inverter, multiplexer, SRAM cells (have zero dynamic power), LUTs, D-Flip-flop and routing switches. The leakage power of each element is state dependent and each state has a probability. The average leakage power of a circuit element can then be written as follows [15]:

$$
P_{\text {avgleak }}=\sum_{i=1}^{n} \text { Prob }_{i} . \text { Pleak }_{i}
$$


where $\operatorname{Prob}_{i}$ and Pleak $k_{i}$ are the probability and leakage power of the state $\mathrm{i}$, and $\mathrm{n}$ is the number of states. Here we assume that the probabilities of the states are equal, i.e., Prob $1=\operatorname{Prob} 2=\ldots=$ Probn $=\frac{1}{n}$. Note that this assumption has no effect on the architectural decisions as the results show.

For example, let us consider an inverter. We have two states: 1) the input is 0 and 2) the input is 1 . With the input at 0 , subthreshold leakage flows through the $\operatorname{NMOS}\left(P_{s u b, n}\right)$ of the inverter and the gate leakage through the PMOS $\left(P_{\text {gate }, p}\right)$. When the input is 1 , there is subthreshold leakage through the PMOS $\left(P_{s u b, p}\right)$ and gate leakage through the NMOS $\left(P_{\text {gate }, n}\right)$. Moreover, in both cases we have BTBT leakage (PBTBT). It should be noted that the leakage values of NMOS and PMOS transistors are extracted from [14]. By assuming an equal probability for both states we have:

$$
P_{\text {leak }}=0.5\left(P_{\text {sub }, n}+P_{\text {gate }, p}+P_{B T B T}\right)+0.5\left(P_{\text {sub }, p}+P_{\text {gate }, n}+P_{B T B T}\right)
$$

On the other hand, the leakage power dissipated in routing network consists of the power of connection boxes, $P_{l k . C B}$, and the power of switch boxes, $P_{l k . S B}$, as expressed in (16). These boxes consist of multiplexers, output buffers and SRAMs. There are two sorts of connection boxes in an FPGA: 1) those connecting routing tracks to CLB inputs and 2) those connecting I/O blocks to routing tracks. These two groups are different because of the different number of inputs to their multiplexers. The leakage power of connection boxes is expressed by (17), where $P_{l k . C B . c l b}$ is the leakage power of the CLB connection box and $P_{l k . C B . i o}$ is the leakage power of the I/O connection box. The weights of Plk.CB.clb and Plk.CB.io in (17) are the number of CLB connection boxes and I/O connection boxes in an FPGA, respectively. $I_{c l b}$ and $I_{i o}$ are the number of CLB and I/O inputs, respectively. There are also two sorts of switch boxes in an FPGA: 1) switching routing tracks in the middle of FPGA and 2) switching tracks at the edge of FPGA where I/O blocks exist. These two groups are different because of the different number of inputs to their multiplexers. The leakage power of switch boxes is expressed by (18), where $P_{l k . S B . e}$ is the leakage power of edge switch box and $P_{l k . S B, m}$ is the leakage power of the middle switch box. The weights of $P_{l k . S B, e}$ and $P_{l k . S B, m}$ in (18) are the number of edge switch boxes and the number of middle switch boxes, respectively. $N_{s, e}$ and $N_{s, m}$ are the number of points in the array where switch boxes occur on the edge and in the center of the grid. Also $1.5 W$ and $2 W$ are the number of channels on the edge and in the center of the grid, respectively. More information about how to compute the number of connection boxes and switch boxes in (17) and (18) can be found in [6].

$$
\begin{aligned}
& P_{l k . r o u t e}=P_{l k . C B}+P_{l k . S B} \\
& P_{l k . C B}=N_{c} I_{c l b} P_{l k . C B . c l b}+4 \sqrt{N_{c}} I_{i o} P_{l k . C B . i o} \\
& P_{l k . S B}=1.5 W N_{s, e} P_{l k . S B, e}+2 W N_{s, m} P_{l k . S B, m}
\end{aligned}
$$

\subsection{Geometric Programming Formulation}

To express model equations discussed above in a form amenable to the GP we need to transform all equations to monomial terms with equality to one, or posynomial terms less than or equal to one. For example the dynamic logic power in (9) is transformed to (19) or the dynamic power of a CLB in (10) is transformed to posynomial inequality in $(20)$.

$$
\begin{aligned}
& N_{c} P_{d y . C L B} P_{d y . l o g i c}{ }^{-1}=1 \\
& K N P_{\text {input.mux }} P_{d y . C L B}{ }^{-1}+N P_{\text {lut }} P_{d y . C L B}{ }^{-1}+N P_{\text {register }} P_{d y . C L B}{ }^{-1}+ \\
& N P_{\text {output.mux }} P_{d y . C L B}{ }^{-1}+K N P_{\text {lut.input.mux }} P_{d y . C L B}{ }^{-1} \leq 1
\end{aligned}
$$

By applying such transformations on the power equations explained in the previous subsections we obtain a complete power model formulated in GP. It is worth noting that our power model in GP can be integrated with the area-delay model from [6] in such a way that a complete performance model in terms of the area, delay and power in GP is obtained. For this purpose, GP format equations from this paper and [6] integrated into a new GP framework (called GP performance model) with a new cost function. The framework consists of three sorts of equations modeling total area, total delay and total power of an FPGA. The equations must be solved subject to minimizing a cost function. The complete model cost function can be represented as (21), which must be minimized. The parameters $z$ and $y$ are between 0 and 1 . By varying $z$ and $y$, we can target the delay $(\mathrm{T})$, area $(\mathrm{A})$ and power $(\mathrm{P})$. For example, setting $z=y=\frac{1}{3}$ lead to equal weighting, or $z=y=0$ will target power.

$$
\text { Minimize : } T^{z} A^{y} P^{1-z-y}
$$




\section{Optimizing Application Specific Architectures in Reconfigurable Computing Mode}

Analytical models accelerate the design of new architectures. For a general-purpose FPGA architecture we would use an analytical model to find optimal values of the architectural parameters. This way, we can use geometric mean of the results from several different benchmark circuits to gain generality. But, most of the time, consumers buy FPGAs for specific application. In this case, designing application-specific FPGAs can significantly improve the performance. Based on a detailed comparison of FPGA and ASIC implementations, it is revealed that the performance (low area, low delay and low power) is lost in FPGAs in the cost of flexibility. On the other hand, ASICs suffer from inflexibility, higher non-recurring engineering (NRE) cost and higher time-to-market. Many solutions have been offered to overcome the disadvantages of FPGA and ASIC implementations. Application Specific Inflexible FPGA (ASIF) is one of such solutions. An ASIF is a reduced FPGA that is able to implement a set of application circuits which will operate at mutually exclusive times [25]. In this section, we have extended the proposed GP performance model in such a way that FPGA architectures can be optimized for a set of applications. The model would be useful in two different ways: 1) from a designer's point of view, to design efficient FPGAs for specific applications like ASIF and 2) from a consumer's point of view, to select the best FPGA device among a variety of existing devices, which is efficient for his or her specific application.

Consider an FPGA will be used for one specific application. Running GP performance model proposed will give us the best values of architectural parameter (including high-level and transistor sizing) for a specific application. In this case, the model equations (expressed in GP format) will be solved to minimize the cost function in (21) where $\mathrm{T}$ is total delay, A is total area and $\mathrm{P}$ is total power.

Now consider an FPGA that will be used for multiple applications, for example an FPGA in a printer may need to implement image processing function in a time and head motor control function in another time. In fact, the device works in a reconfigurable computing mode. The device is reconfigured to proper function needed, many times. We will develop GP performance model to find the best values of architectural parameters when targeting a set of applications for an FPGA. In this paper we investigate a double function case, but the approach is general that could be used for any number of functions.

For a double function case the cost function in (21) is used where area, delay and power of the architecture can be expressed as (22), (23) and (24), where $A_{1}$ and $A_{2}$ are the areas of the first circuit (circuitl) and the second one (circuit2), respectively. $T_{1}$ and $T_{2}$ are the delays of circuitl and circuit 2 respectively. The dynamic powers of circuit 1 and circuit 2 can be represented by $P_{d y 1}$ and $P_{d y 1} . P_{l k}$ is the leakage power of the architecture and would be defined next.

$$
\begin{aligned}
& A_{\text {tot }}=\max \left(A_{1}, A_{2}\right) \\
& T_{t o t}=f_{1} T_{1}+f_{2} T_{2} \\
& P_{t o t}=P_{l k}+f_{1} P_{d y 1}+f_{2} P_{d y 2}
\end{aligned}
$$

Consider an FPGA architecture, which would be used to implement two circuits in different times. The suitable area of architecture must be selected as the maximum area consumption of circuits when mapping into architecture. The delay can be selected as the weighted addition of delays when the weights are proportional to the time of each circuit implemented. So $f_{1}$ in (23) can be defined as (25) and $f_{2}$ is the complement of $f_{1}$ i.e. $f_{2}=1-f_{1}$. It is obvious that $f_{1}$ and $f_{2}$ are between 0 and 1 . For example setting $f_{1}=0.75$ and $f_{2}=0.25$ means that the FPGA would implement circuit 1 in $75 \%$ of the time and circuit 2 in remaining $25 \%$. The power must be divided into two parts: leakage and dynamic. The leakage power is mainly dependent to architecture itself and not to the function so the leakage power can be selected as the maximum leakage power of circuits when mapping into architecture as in (26). The dynamic power is mainly dependent to the function which is implemented on architecture so it can be added to the total power as in (24) where factors are discussed before.

$$
\begin{aligned}
& f_{1}=\frac{\text { Percent of overall time that circuit } 1 \text { works }}{\text { Overal time of system } \text { working }} \\
& P_{l k}=\max \left(P_{l k 1}, P_{l k 2}\right)
\end{aligned}
$$

To complete the framework the model equations including area equations, delay equations and power equations must be rewritten for both circuits. Therefore, we have two sets of equations: 1) one set to calculate circuitl's area $\left(A_{1}\right)$, delay $\left(T_{1}\right)$, power $\left(P_{l k 1}, P_{d y 1}\right)$ and 2) another set to calculate circuit2's area $\left(A_{2}\right)$, delay $\left(T_{2}\right)$, power $\left(P_{l k 2}, P_{d y 2}\right)$. There are also two groups of parameters in the model: 1) architectural parameters which are describing the 
architecture and 2) circuit parameters which are describing the circuits. The architectural parameters, such as cluster size (N) or channel width (W) would be the same in both sets of equations, because the architecture is unique. The circuit parameters, such as the number of 2-LUTs in 2-LUT implementation, are different for each set of equation. Each circuit has its own circuit parameters in equations. For example, the number of 2-LUTs of circuitl is $n_{2,1}$ while the number of 2-LUTs of circuit2 is $n_{2,2}$.

For example, consider the delay equation of the critical signal path through a circuit implemented on an FPGA. This equation is shown in (5). The critical path passes through a number of circuit components. Each component has a delay. The critical path delay is modeled as a weighted sum of various components' delay as shown in (5). Each component's delay depends on resistance along the path to the component and output capacitance of component so it is an architectural parameter. On the other hand, the weights in (5) are the number of each component along the path and are depending on depth of circuit so they are circuit parameters.

The critical path delay equations for circuitl and circuit 2 in the double function case of our model can be written as (27) and (28).

$$
\begin{gathered}
T_{\text {total } 1}=T_{\text {reg to oDrv }}+D_{i 1} T_{L U T \text { F } / B \text { path }}+\left(D_{k 1}-1\right) T_{L U T \text { delay }}+D_{c 1} T_{\frac{o}{P} C B \text { delay }}+D_{c 1} D_{r 1} T_{S B \text { delay }}+ \\
D_{c 1} T_{\frac{I}{P} C B \text { delay }}+D_{c 1} T_{\text {input } M U X \text { delay }}+T_{\text {LUT to reg delay }} \\
T_{\text {total } 2}=T_{\text {reg to } O D r v}+D_{i 2} T_{L U T F / B \text { path }}+\left(D_{k 2}-1\right) T_{L U T \text { delay }}+D_{c 2} T_{\frac{o}{P} C B \text { delay }}+D_{c 2} D_{r 2} T_{S B \text { delay }}+ \\
D_{c 2} T_{\frac{I}{P} C B \text { delay }}+D_{c 2} T_{\text {input } M U X \text { delay }}+T_{L U T \text { to reg delay }}
\end{gathered}
$$

It should be noted that the architectural parameters have been selected the same in both sets of equations, because the architecture is unique while the circuit parameters are different for each set of equations, because the circuits are different.

\section{$5 \quad$ Experimental Results}

\subsection{Power Model Verification}

In order to illustrate the effectiveness of the proposed model and evaluate its accuracy we have performed several experiments. We have employed $10 \mathrm{MCNC}$ benchmark circuits, including ex5p, misex3, apex4, alu4, tseng, seq, apex2, diffeq, dsip and des, to evaluate the accuracy of the proposed power model. Comparison between GP power model and the VPR power model [10] is done in all cases. Moreover, we have used CVX optimization tool [23] to solve the GP equations. Although we make use of $65 \mathrm{~nm}$ technology parameters of Predictive Technology Model (PTM) [19], the model is general enough to be applied to any other technology.

Fig. 6(a) shows the geometric mean of the total power across the circuits when sweeping the cluster size (N). Each data point is also the geometric mean of the results for LUT size $(\mathrm{K})$ of 2-7. Although the results obtained from our model track those of the VPR power model, magnitudes are different due to different technologies used. VPR power model is using $180 \mathrm{~nm}$ technology parameters. In all of runs we have taken into account a constant clock frequency at the value of 20MHZ, so the power behavior would be the same as the energy. In fact, speed is one of the parameters to be optimized in the model, so its value must be fixed after optimization. Here we have used the example value of $20 \mathrm{MHz}$ to express the optimization parameter as the power (and not the energy). So this value is not important and has no effect on the optimization process.

Fig. 6(b) shows the geometric mean of the total power across the circuits when sweeping the LUT size (K). Each data point is also the geometric mean of the results for cluster size $(\mathrm{N})$ of 2-20. To further investigation, the dynamic and leakage powers consumed by the logic parts as well as the routing paths based on the proposed power model and VPR power model have been illustrated in Fig. 7. Each data point is the geometric mean across the circuits and cluster size $(\mathrm{N})$ of 2-20.

The increase of leakage power when technology shrinks from $180 \mathrm{~nm}$ to $65 \mathrm{~nm}$ can be seen in Fig. 7(b) and Fig. 7(d). It is obvious that the leakage power of the routing part increases by $\sim 4 \mathrm{X}$ and the leakage power of the logic part increases by $\sim 12 \mathrm{X}$. It should be noted that our proposed power model considers the gate leakage power and BTBT leakage power while VPR power model does not. As can be seen in Fig. 7(c), the results of Davis/Lam wirelength model that is chosen to model the routing dynamic power, match those of VPR power model in a good 
manner. Please note that, although we make use of the VPR power model to validate the routing dynamic power based on Davis/Lam wire-length model in subsection 3.1.1, the effect of technology resizing is not taken into account in Fig 7(c). Fig. 8 also shows the dynamic and leakage powers consumed by the logic parts as well as the routing paths based on the proposed power model and VPR power model, when sweeping the cluster size (N). Each data point is the geometric mean across the circuits and LUT size (K) of 2-7. Using LUTs with more inputs and CLBs with greater size reduce the total number of LUTs and CLBs required for mapping a circuit on architecture. This reduces the routing network power and increases the logic part power. This effect can be seen in Fig 7 and Fig 8.

To show the effectiveness of our GP power model in terms of the run-time, we have measured the run-time of both VPR power model and GP framework. The result is performed on a $2.2 \mathrm{GHz}$ DualCore with $2 \mathrm{~GB}$ of RAM running on Linux. The run-time of VPR power model for a sweep across $K=2-7$ and $N=2-20$, by the step of two, is 90 minutes against 10 minutes for our GP model, i.e. $9 \times$ run time improvement. Note that the VPR power model needs to complete placement and routing to report the power; while the GP model is reporting power simply by optimizing the analytical model. This is the main reason of run time improvement for the analytical methods of FPGA architecture optimization, compared to the experimental ones.

\subsection{GP Performance Model Verification}

Each part of GP performance model has been verified previously. To verify the GP performance model integration, comparison with experimental results is made. The results of GP performance model are shown in Fig 9(a), while Fig 9(b) shows the results collected from VPR (area), HSPICE (delay), and VPR power model. The VPR would report the consumed area. The delay can be calculated by simulating each path of critical path from equation (5) in transistor level in HSPICE and measure the delay time between 50\% rise of input step signal to 50\% rise/fall of output; after that, by applying the weights of equation (5) the critical path delay will be calculated.

What we would like to present in Fig. 9 is the value of cost function of $(21)(z=y=1 / 3)$ when the architectural parameters are changed. This way, we are able to find the optimized architectural parameters. In Fig. 9 two parameters of $\mathrm{K}$ and $\mathrm{N}$ are investigated. To show cost values versus two parameters of $\mathrm{K}$ and $\mathrm{N}$ contours have been employed. The contours show the minimum value where the lines running through the location of. The minimum value would introduce the optimal values of architectural parameters of $\mathrm{K}$ and N. Fig. 9(a) shows the geometric mean of the area-delay-power product across the circuits when sweeping the cluster size (N) and the LUT size (K). Area, delay and power are weighted as $z=y=1 / 3$ in equation (21) i.e. area, delay and power have equal importance. Similarly Fig. 9(b) shows the area-delay-power product when power data are extracted from VPR power model. It is obvious that the same architectural conclusions would be made from both contours. Both contours show 5 as the optimal value of $\mathrm{K}$ and 6-7 as the optimal value of $\mathrm{N}$.

\subsection{Performance Model for FPGA-based Reconfigurable Computing}

To show the results of the model developed for a set of applications we have performed some experiments. The CVX optimization tool has been used to solve the GP formatted equations with proper cost function. More details have been described in Section 4. First we have run GP performance model for 2 MCNC benchmark circuits. Fig. 10(a) and Fig. 10(b) show the area-delay-power product across the apex4 and bigkey benchmark circuits when sweeping the cluster size $(\mathrm{N})$ and the LUT size $(\mathrm{K})$. The circuits offer different architectures by different cluster size. The optimal value of cluster size $(\mathrm{N})$ is 8 for apex 4 and 4 for bigkey.

In the next step, we have run the developed multi-application model to find the optimized architecture for the set of apex 4 and bigkey. Fig. 10(c) shows the area-delay-power product for the architecture when $f_{1}$ factor is 0.75 $\left(f_{2}=0.25\right)$, i.e. 75 percent of the times the system needs to implement apex 4 and 25 percent of the times the system needs to implement bigkey. Fig. 10(d) shows the area-delay-power product for the architecture when $f_{1}$ factor is 0.5 $\left(f_{2}=0.5\right)$ i.e. the system equally implement apex 4 and bigkey at exclusive times. Fig. 10(e) shows the area-delaypower product for the architecture when $f_{1}$ factor is $0.25\left(f_{2}=0.75\right)$.

Fig. 10 shows the optimal parameters migrate from apex 4 optimal parameters to bigkey optimal parameters when $f_{1}$ factor varied from 0 to 1 (or $f_{2}$ factor varied from 1 to 0 ) as expected. Table 2 gives the results of this experiment including total area, delay (T1: delay for $\mathrm{f} 1, \mathrm{~T} 2$ : delay for $\mathrm{f} 2$ and Ttot: total delay), power (Plk: leakage power, Pdy1: dynamic power for f1, Pdy2: dynamic power for $\mathrm{f} 2$ and Ptot: total power) and cost values for each 
architecture with optimal values for $\mathrm{K}$ and $\mathrm{N}$. Note that the cost value is from (21) in which $z=y=1 / 3$. The results show different architectures for apex4 (Fig. 10(a)) and bigkey (Fig. 10(b)) in terms of cluster size (N). The optimal value of $\mathrm{N}$ for the combinations of apex4 and bigkey with different working factors (Fig. 10(c,d,e)) are varying between two circuits' optimal value of $\mathrm{N}$.

\section{Conclusion}

This paper has presented a complete analytical performance model for FPGAs. The model consists of area, delay and power model. A method to analytically estimate both dynamic and static power of FPGAs has described in detail. Integration of the power model proposed in this paper with the area and delay model of [6] allows designers a fast exploration of FPGA architecture parameters. Our results show that up to $9 \mathrm{X}$ run time improvement in comparison with the VPR based power model is achieved. By using the GP framework, concurrent optimization of high-level and low-level parameters is obtainable. After integrating the power model into GP performance model, the model developed for optimizing reconfigurable computing architectures. This way, FPGA architecture will reconfigure between a set of applications. A case of an architecture which is implementing two circuits investigated.

In the future, we intend to work more on the run time of analytical models compared to experimental methods. Possible other frameworks than geometric programming will be investigated. Automation of analytical methods to explore FPGA architectures is another interesting path.

\section{Acknowledgment}

This research was in part supported by Iran National Science Foundation (INSF) under grant \#92018585.

\section{REFERENCES}

1. Das, J. and Wilton, S.J.E.: Towards development of an analytical model relating FPGA architecture parameters to routability. ACM Transactions on Reconfigurable Technology and Systems (TRETS) 6, 2, Article 10, 24 pages (2013)

2. Fang, W.M. and Rose, J.: Modeling routing demand for early-stage FPGA architecture development. Proceedings of the 16th international ACM/SIGDA symposium on Field programmable gate arrays, pp.139-148 (2008)

3. Mehri, H. and Alizadeh, B.: An Analytical Dynamic and Leakage Power Model for FPGAs. Proceedings of the $22^{\text {nd }}$ Iranian conference on electrical engineering, pp. 300-305 (2014)

4. Das, J., Lam, A., Wilton, S. J. E., Leong, P. and Luk, W.: An Analytical Model Relating FPGA Architecture to Logic Density and Depth. IEEE Transactions on Very Large Scale Integration Systems, vol. 19, no. 12, pp. 2229-2242 (2010)

5. Smith, A.M., Das, J. and Wilton, S.J.E.: Wirelength modeling for homogeneous and heterogeneous FPGA architectural development. Proceedings of the ACM/SIGDA international symposium on Field programmable gate arrays, pp. 181-190 (2009)

6. Smith, A.M., Constantinides, G. and Cheung, P.: FPGA architecture optimization using geometric programming. IEEE Transactions on Computer-Aided Design of Integrated Circuits and Systems, vol. 29, no. 8, pp. 1163 -1176 (2010)

7. Smith, A.M., Constantinides, G. and Cheung, P.: Area estimation and optimization of FPGA routing fabrics. Proceedings of Field Programmable Logic and Applications, pp. 256-261 (2009)

8. Hung, E., Yu, H., Chau, T., Leong, P.H.W. and Wilton, S.J.E.: A Detailed Delay Path Model for FPGAs. Proceedings of Field Programmable Technology, pp. 96-103 (2009)

9. Smith, A. M., Constantinides, G. A., Wilton, S. J. E. and Cheung, P. Y. K.: Concurrently optimizing FPGA architecture parameters and transistor sizing: Implications for FPGA design. Proceedings of Field Programmable Technology, pp. 54-61 (2009)

10. Poon, K. W., Wilton, S. J. E. and Yan, A.: A detailed power model for field-programmable gate arrays. ACM Trans. Des. Autom. Electron. Syst. 10, 2, pp. 279-302 (2005)

11. Li, F., Lin, Y., He, L., Chen, D. and Cong, J.: Power modeling and characteristics of field programmable gate arrays. Trans. Comp.-Aided Des. Integ. Cir. Sys. 24, 11, 1712-1724 (2006)

12. Lin, Y., Li, F. and He, L.: Power modeling and architecture evaluation for FPGA with novel circuits for Vdd programmability. In Proceedings of the 2005 ACM/SIGDA 13th international symposium on Field-programmable gate arrays. ACM, New York, NY, USA, 199-207 (2005) 
13. Gulati, K., Khatri, S.P. and Li, P.: Closed-loop modeling of power and temperature profiles of FPGAs. In Proceedings of the ACM/SIGDA international symposium on Field programmable gate arrays. ACM, New York, NY, USA, 287-287 (2009)

14. Rastogi, A., Chen, W., Sanyal, A. and Kundu, S.: An Efficient Technique for Leakage Current Estimation in Sub 65nm Scaled CMOS Circuits Based on Loading Effect. In Proceedings of the 20th International Conference on VLSI Design held jointly with 6th International Conference: Embedded Systems. IEEE Computer Society, Washington, DC, USA, 583-588 (2007)

15. Kumar, A. and Anis, M.: An analytical state dependent leakage power model for FPGAs. In Proceedings of the conference on Design, automation and test in Europe. European Design and Automation Association, 3001 Leuven, Belgium, Belgium, 612-617 (2006)

16. Rajavel, S.T. and Akoglu, A.: An analytical energy model to accelerate FPGA logic architecture investigation. In Proceedings of Field Programmable Technology. 1-8 (2011)

17. Parvez, H. and Mehrez, H.: Application-Specific Mesh-based Heterogeneous FPGA Architectures. New York: Springer, ch. $1(2011)$

18. Boyd, S.P., Kim, S.J., Patil, D. and Horowitz, M.A.: Digital Circuit Optimization via Geometric Programming. Oper. Res. 53, 6, 899-932 (2005)

19. Predictive Technology Model 2011, http://ptm.asu.edu/

20. Singh, A. and Sadowska, M.: Efficient circuit clustering for area and power reduction in FPGAs. In Proceedings of the 2002 ACM/SIGDA tenth international symposium on Field-programmable gate arrays. ACM, New York, NY, USA, 59-66 (2002)

21. Luu, J., Kuon, I., Jamieson, P., Campbell, T., Ye, A., Fang, W.M., Kent, K. and Rose, J.: VPR 5.0: FPGA CAD and architecture exploration tools with single-driver routing, heterogeneity and process scaling. ACM Trans. Reconfigurable Technol. Syst. 4, 4, Article 32, 23 pages (2011)

22. Betz, V., Rose, J. and Marquardt, A.: Architecture and CAD for Deep-Submicron FPGAs. Kluwer Academic Publishers, Norwell, MA, USA (1999)

23. Grant, M. and Boyd, S.: CVX: Matlab software for disciplined convex programming, version 2.0 beta. http://cvxr.com/cvx (2012)

24. Das, J., Wilton, S.J.E.: Accelerated FPGA Architecture Design: Capabilities and Limitations of Analytical Models. In Proceedings of International Conference on Field Programmable Technology. 1-8 (2011)

25. Parvez, H., Marrakchi, Z., and Mehrez, H.: ASIF: Application Specific Inflexible FPGA. In Proceedings of International Conference on Field Programmable Technology. 112-119 (2009)

26. Parvez, H., Marrakchi, Z. and Kilic, A. and Mehrez, H.: Application-Specific FPGA using heterogeneous logic blocks. ACM Trans. Reconfigurable Technol. Syst. 4, 3, Article 24, 14 pages (2011)

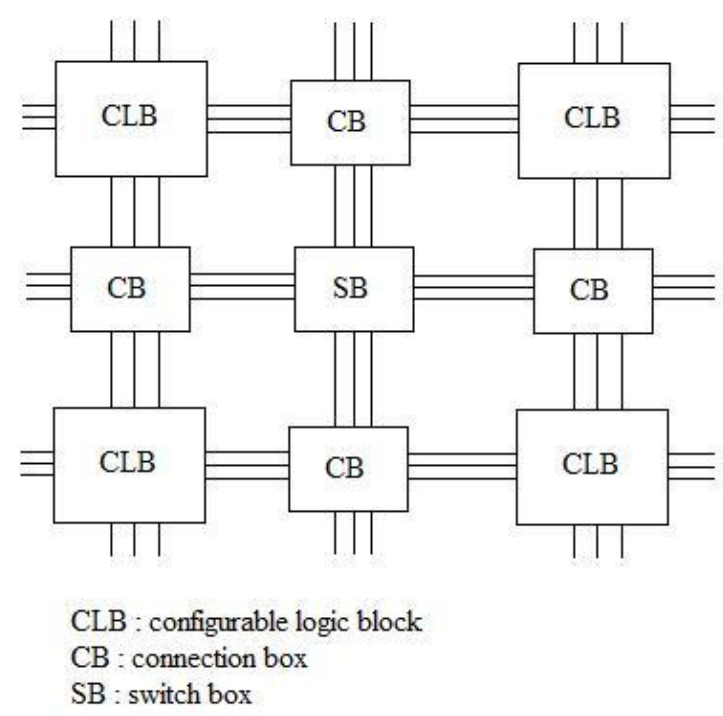

Fig. 1. Mesh-based FPGA internal architecture. 


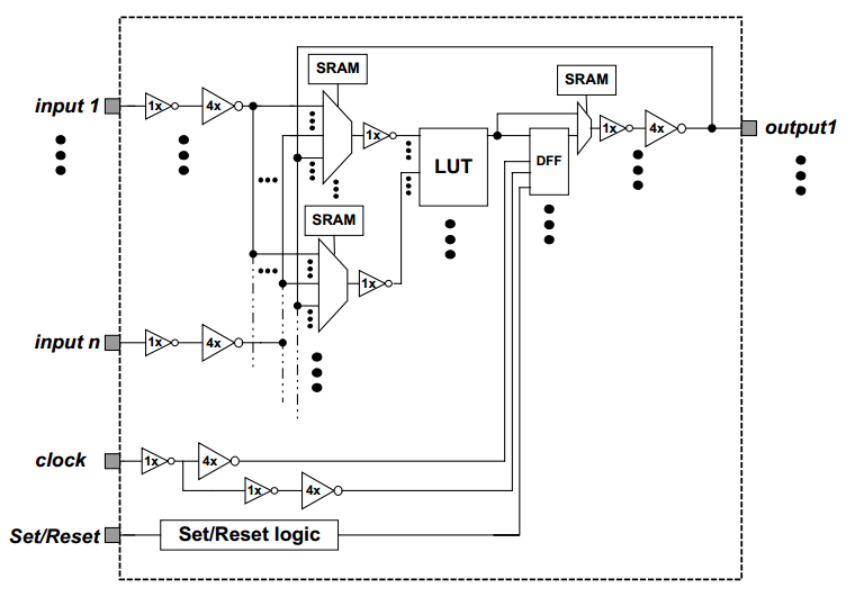

Fig. 2. Internal structure of a CLB (from [22]).

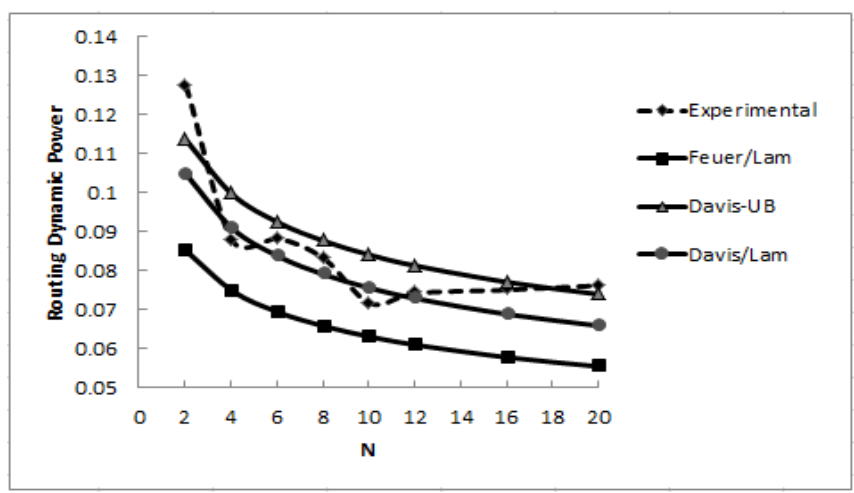

Fig. 3. Routing dynamic power modeling using wire-length models.

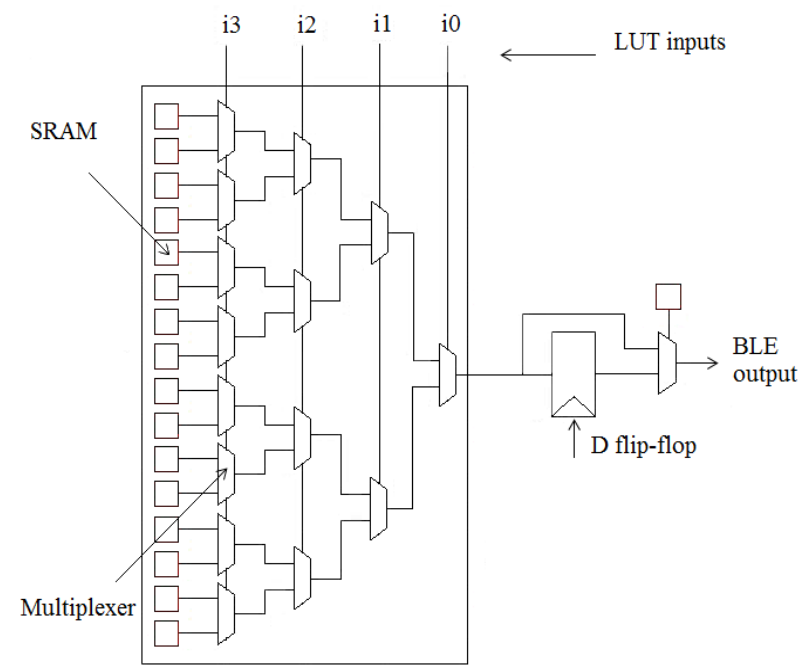

Fig. 4. A 4-input LUT structure. 


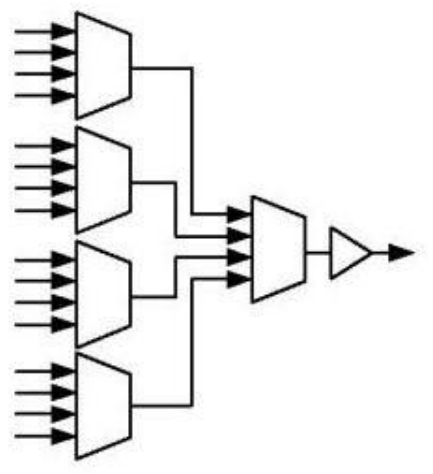

(a)

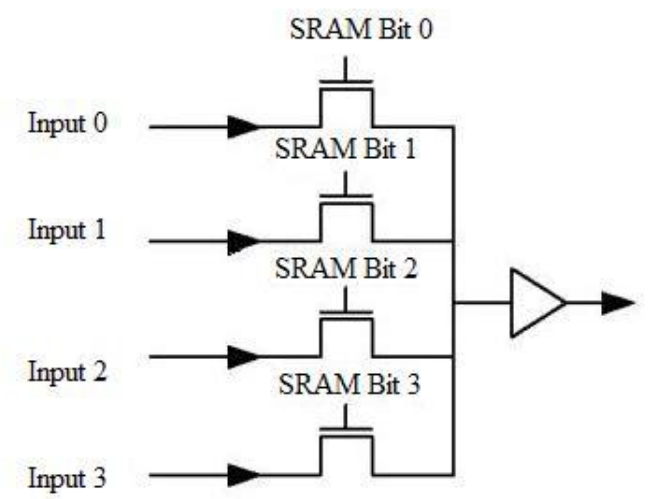

(b)

Fig. 5. Multiplexer structure in VPR 5.0 : (a) a two-level 16:1 multiplexer, (b) a pass transistor based 4:1 multiplexer (from [6]).

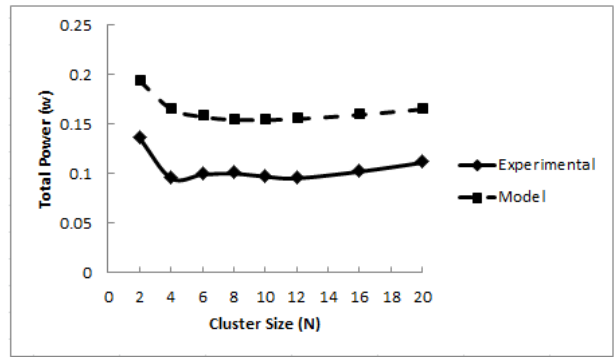

(a)

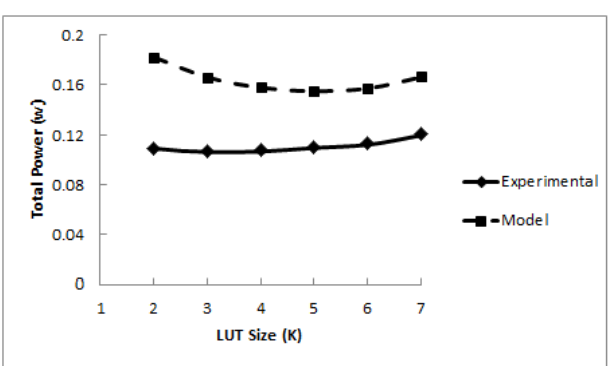

(b)

Fig. 6. Comparing proposed power model with VPR power model: (a) variation of N, (b) variation of K.

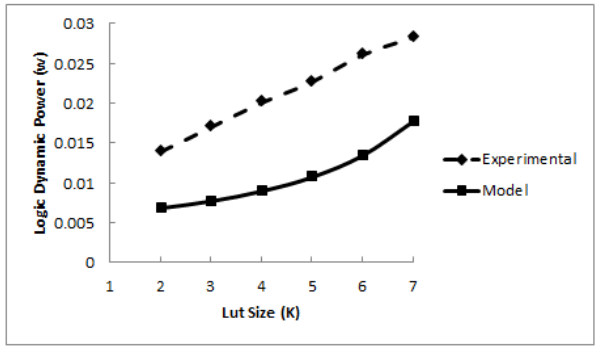

(a)

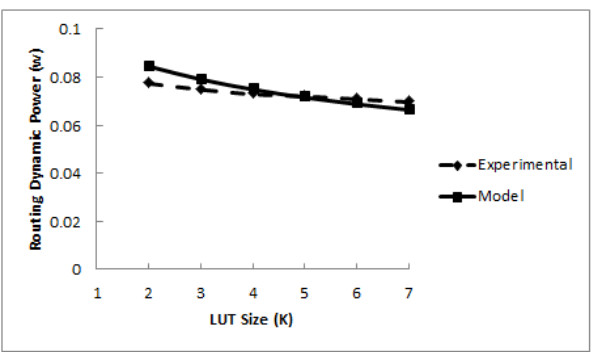

(c)

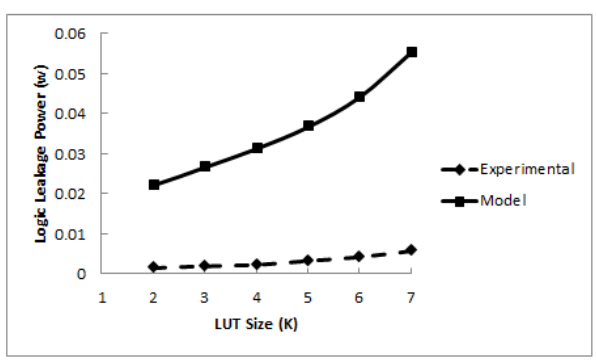

(b)

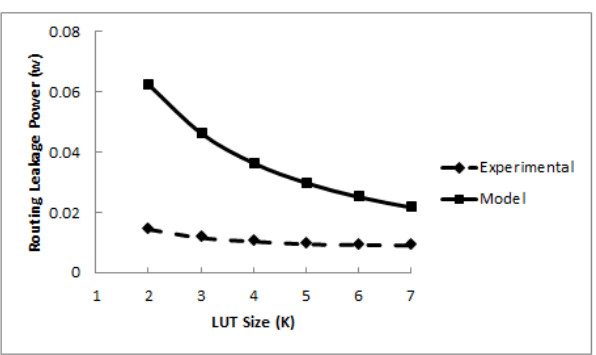

(d)

Fig. 7. Each part of power when K varied: (a) logic blocks dynamic power, (b) logic blocks leakage power, (c) routing network dynamic power, (d) routing network leakage power. 

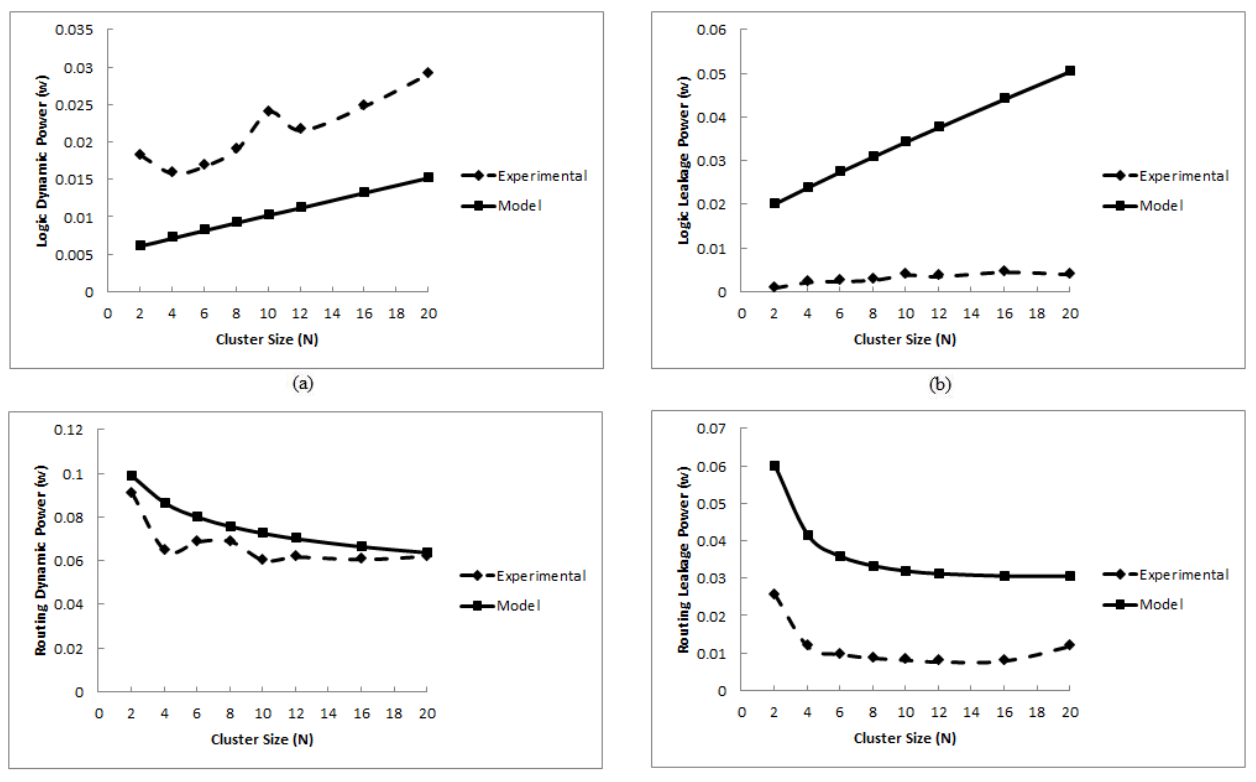

(c)

(d)

Fig. 8. Each part of power when $\mathrm{N}$ varied: (a) logic blocks dynamic power, (b) logic blocks leakage power, (c) routing network dynamic power, (d) routing network leakage power.

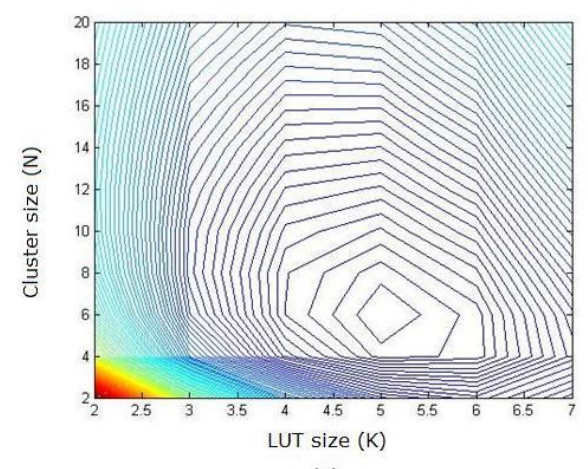

(a)

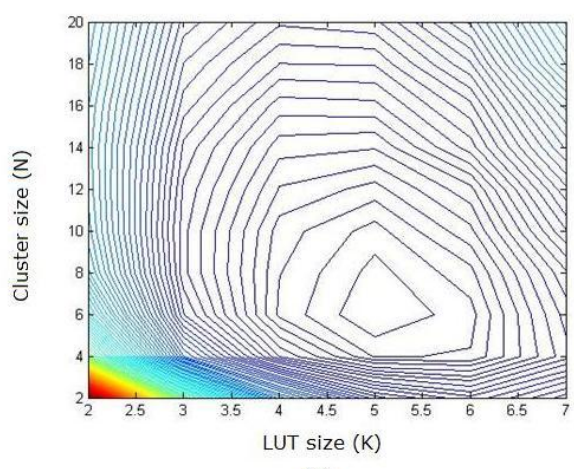

(b)

Fig. 9. Comparing GP performance model with experimental results: (a) GP results, (b) experimental results. 


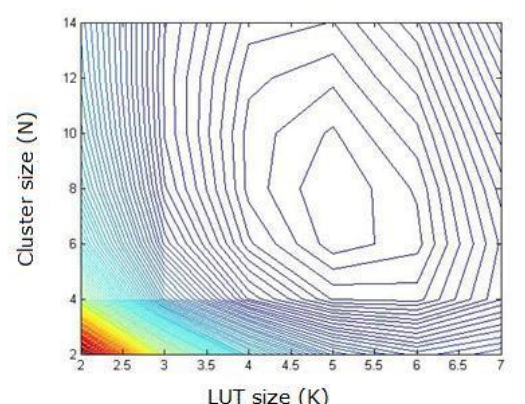

(a)

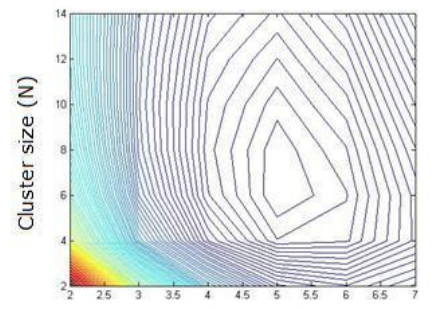

LUT size (K)

(c)

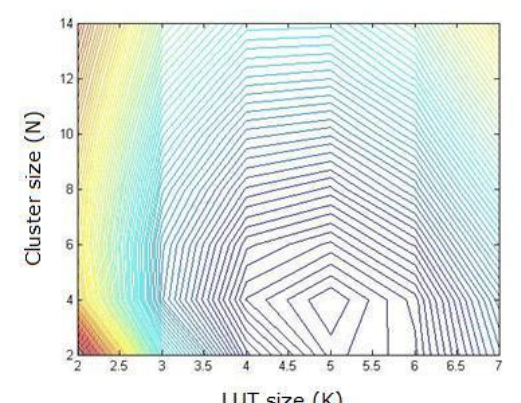

(b)

Fig. 10. Results from GP performance model and developed double-application model for two circuits: (a) optimized architecture for apex4, (b) optimized architecture for bigkey. Optimized architecture for double-application of apex4 and bigkey: (c) when $f_{1}=0.75$ and $f_{2}=0.25$, (d) when $f_{1}=f_{2}=0.5$, (e) when $f_{1}=0.25$ and $f_{2}=0.75$.

Table 1. Model Parameters

\begin{tabular}{|c|c|}
\hline \multicolumn{2}{|c|}{ Architectural Parameters (high-level): } \\
\hline $\mathrm{N}$ & Cluster Size \\
\hline $\mathrm{K}$ & Lookup Table Size \\
\hline I & Inputs per Cluster \\
\hline $\mathrm{Fc}_{\text {in/out }}$ & Connection box flexibility \\
\hline Fs & Switch box flexibility \\
\hline \multicolumn{2}{|c|}{ Low-level Parameters: } \\
\hline $\mathrm{S}_{\mathrm{n}, 21 \mathrm{mux}}$ & Scaling of pass transistors in the $2: 1 \mathrm{mux}$ \\
\hline $\mathrm{S}_{\mathrm{n}, \mathrm{LM}}$ & Scaling of pass transistors in the LUT mux \\
\hline $\mathrm{S}_{\mathrm{n}, \mathrm{SB}}$ & Scaling of pass transistors in the switch box mux \\
\hline $\mathrm{S}_{\mathrm{n}, \mathrm{CB}}$ & Scaling of pass transistors in the connection box mux \\
\hline $\mathrm{S}_{\mathrm{n} / \mathrm{p}, \text { LIdrv }}$ & Scaling of $\mathrm{n} / \mathrm{pMOS}$ transistor in LUT input driver \\
\hline $\mathrm{S}_{\mathrm{n} / \mathrm{p}, \text { LOdrv }}$ & Scaling of $\mathrm{n} / \mathrm{pMOS}$ transistor in LUT output driver \\
\hline $\mathrm{S}_{\mathrm{n} / \mathrm{p}, \mathrm{SBdrv}}$ & Scaling of $\mathrm{n} / \mathrm{pMOS}$ transistor in switch box driver \\
\hline $\mathrm{S}_{\mathrm{n} / \mathrm{p}, \mathrm{CB} d \mathrm{drv}}$ & Scaling of $\mathrm{n} / \mathrm{pMOS}$ transistor in connection box driver \\
\hline \multicolumn{2}{|c|}{ Circuit Parameters: } \\
\hline $\mathrm{n}_{2}$ & Number of 2-LUTs for 2-LUT implementation \\
\hline $\mathrm{d}_{2}$ & Depth of circuit for 2-LUT implementation \\
\hline $\mathrm{p}$ & Rent parameter of circuit \\
\hline
\end{tabular}


Table 2. Area, delay, power and cost values for optimal architecture of experiments when $f_{1}$ and $f_{2}$ varies (Area is given in the number of transistors; Delay is given in seconds; Power is given in w; NA: Not applicable)

\begin{tabular}{|c|c|c|c|c|c|c|}
\hline \multicolumn{2}{|c|}{$\begin{array}{l}f_{1}: \text { apex } 4 \\
f_{2} \text { :bigkey }\end{array}$} & $\begin{array}{l}f_{1}=1 \\
f_{2}=0\end{array}$ & $\begin{array}{l}f_{1}=0.75 \\
f_{2}=0.25\end{array}$ & $\begin{array}{l}f_{1}=0.5 \\
f_{2}=0.5\end{array}$ & $\begin{array}{l}f_{1}=0.25 \\
f_{2}=0.75\end{array}$ & $\begin{array}{l}f_{1}=0 \\
f_{2}=1\end{array}$ \\
\hline \multicolumn{2}{|c|}{ Area } & $1.8461 \mathrm{e}+006$ & $1.7260 \mathrm{e}+006$ & $1.5958 \mathrm{e}+006$ & $1.4479 \mathrm{e}+006$ & $\begin{array}{c}1.2392 \mathrm{e}+00 \\
6\end{array}$ \\
\hline \multirow{3}{*}{ Delay } & $\mathrm{T}_{1}$ & $2.8134 \mathrm{e}-009$ & $2.8237 \mathrm{e}-009$ & $2.8388 \mathrm{e}-009$ & $2.8614 \mathrm{e}-009$ & NA \\
\hline & $\mathrm{T}_{2}$ & NA & $1.8773 \mathrm{e}-009$ & $1.8567 \mathrm{e}-009$ & $1.8341 \mathrm{e}-009$ & $1.8075 \mathrm{e}-009$ \\
\hline & $\mathrm{T}_{\text {tot }}$ & $2.8134 \mathrm{e}-009$ & $2.5871 \mathrm{e}-009$ & $2.3477 \mathrm{e}-009$ & $2.0909 \mathrm{e}-009$ & $1.8075 \mathrm{e}-009$ \\
\hline \multirow{4}{*}{ Power } & $\mathrm{P}_{\mathrm{lk}}$ & 0.0709 & 0.0656 & 0.0599 & 0.0535 & 0.0444 \\
\hline & $\mathrm{Pdy}_{1}$ & 0.0889 & 0.0907 & 0.0929 & 0.0957 & NA \\
\hline & $\mathrm{Pdy}_{2}$ & NA & 0.0741 & 0.0750 & 0.0763 & 0.0780 \\
\hline & $P_{\text {tot }}$ & 0.1598 & 0.1522 & 0.1439 & 0.1346 & 0.1224 \\
\hline \multicolumn{2}{|c|}{ Cost } & 0.0939766 & 0.0879143 & 0.0813871 & 0.0741348 & 0.0649593 \\
\hline
\end{tabular}

